\title{
A criação de sítio para disseminar informações sobre medicamentos no SUS: um relato de experiência
}

\section{Creating site to disseminate information about medicines in the SUS: an experience report}

\section{Creación de sitio para difundir información acerca de los medicamentos en el SUS: un relato de experiencia}

\author{
Caroline de Godoi Rezende Costa Molino | carolgodoi@gmail.com \\ Universidade de São Paulo, Faculdade de Ciências Farmacêuticas, Departamento de Farmácia. São Paulo, Brasil. \\ Daniela Oliveira Melo | melo.daniela@unifesp.br \\ Universidade Federal de São Paulo, Instituto de Ciências Ambientais, Químicas e Farmacêuticas, Departamento de \\ Ciências Biológicas. São Paulo, Brasil. \\ Eliane Ribeiro | eribeiroo@gmail.com \\ Universidade de São Paulo, Faculdade de Ciências Farmacêuticas, Departamento de Farmácia. São Paulo, Brasil.
}

\section{Resumo}

Neste estudo é relatado o processo de desenvolvimento de sítio sobre medicamentos no SUS. Para a escolha dos temas a serem inseridos no sítio, primeiramente foram elencadas as dificuldades de entendimento quanto a Assistência Farmacêutica apresentada pelos profissionais de saúde e usuários do SUS. Em seguida, estes problemas foram discutidos com farmacêuticos especialistas para delimitação dos tópicos e conteúdo para criação das páginas no sítio. Os tópicos incluídos estão divididos em 2 grandes temas: informações sobre a Assistência Farmacêutica no SUS e orientações para a promoção do uso racional de medicamentos. O processo de desenvolvimento do sítio pode servir como exemplo para outros programas de residência, contribuindo para realização de trabalhos de conclusão de residência que disseminem informações relevantes para o usuário do SUS (profissional de saúde ou paciente) na internet e favoreçam a integração entre serviço, ensino, pesquisa e comunidade.

Palavras-chave: assistência farmacêutica; disseminação de informação; comunicação em saúde; internet; residências em farmácia. 


\begin{abstract}
The aim of this study was to report the process of a website creation that disseminates information on SUS drugs. First, topics for the website were selected based on problems in understanding the Pharmaceutical Service presented by healthcare providers and patients. Then these problems were discussed with pharmaceutical specialists to determine the topics and content to be included on the website. The topics were divided into two mains themes: information on the Pharmaceutical Service in SUS, and guidelines to promote the rational use of medicines. The website development report may serve as an example for other residency programs, and may contribute to the development of other residency theses that disseminate relevant information to patients and healthcare providers, promoting the integration of service, teaching, research and community.
\end{abstract}

Keywords: pharmaceutical services; information dissemination; health communication; internet; pharmacy residencies.

\title{
Resumen
}

El objetivo de este estudio fue describir el proceso de creación de un sitio web que difunde información sobre las drogas SUS. En primer lugar, fueron seleccionados los temas para el sitio basado en problemas en la comprensión del Servicios Farmacéuticos presentado por los profesionales sanitarios y los pacientes. Entonces estos problemas se discutieron con especialistas farmacéuticos para delimitación de los temas y el contenido para crear las páginas en el sitio. Los temas fueron divididos en dos principales temas: información sobre el Servicios Farmacéuticos en el SUS, y directrices para promover el uso racional de los medicamentos. El proceso de desarrollo del sitio puede servir de ejemplo para otros programas de residencia, y puede contribuir al desarrollo de otras tesis de residencia que difunden información relevante para los pacientes y los profesionales de la salud, la promoción de la integración de los servicios, la enseñanza, la investigación y la comunidad.

Palabras clave: servicios farmacéuticos; diseminación de información; comunicación en salud; internet; residencias en farmacia.

Contribuição dos autores: Todas os autores contriburam significantemente para o trabalho;

Declaração de conflito de interesses: não há;

Fontes de financiamento: não há;

Agradecimento/Contribuições adicionais: não há;

Histórico do artigo: Submetido: 06.out.2016 | Aceito: 23.fev.2017 | Publicado: 31.mar.2017

Apresentação anterior: Nenhuma;

Licença CC BY-NC atribuição não comercial. Com essa licença é permitido acessar, baixar (download), copiar, imprimir, compartilhar, reutilizar e distribuir os artigos, desde que para uso não comercial e com a citação da fonte, conferindo os devidos créditos de autoria e menção à Reciis. Nesses casos, nenhuma permissão é necessária por parte dos autores ou dos editores. 


\section{Introdução}

O Sistema Único de Saúde (SUS) foi instituído pela Constituição de 1988 e baseia-se nos seguintes princípios: universalidade, integralidade e participação social ${ }^{1}$. O SUS é financiado por impostos e contribuições sociais e fornece cuidados de saúde gratuitos em nível primário, secundário e terciário².

Ao longo dos anos, o SUS tem criado, implementado e investido em políticas de saúde que melhoraram o acesso aos medicamentos ${ }^{3-5}$. No entanto, ainda há uma grande dificuldade em compreender a complexa estruturação da Assistência Farmacêutica no SUS, não só pelos pacientes, mas também pelos profissionais de saúde: qual e onde estão os formulários exigidos, onde o medicamento pode ser encontrado, e quais os documentos que os pacientes devem apresentar ${ }^{3}$.

$\mathrm{Na}$ atenção primária é comum que o usuário não consiga adquirir o medicamento, entre outros fatores, devido ao preenchimento incorreto da prescrição, ausência de dados necessários a dispensação e compreensão da posologia, como por exemplo: data, tempo de tratamento, assinatura ou carimbo do prescritor, dose/ apresentação farmacêutica, medicamento com nome comercial, letra ilegível e prescrição de medicamento não padronizado na rede pública ${ }^{6-11}$. Problemas nas prescrições somadas ao baixo nível socioeconômico dos pacientes brasileiros favorecem o risco de desfechos negativos ${ }^{12}$ que aumentam a demanda pelos serviços de saúde, frequentemente exigindo níveis mais complexos de cuidado, trazendo um aumento desnecessário dos gastos em saúde e reduzindo a qualidade de vida dos pacientes ${ }^{13}$.

Além disso, a não compreensão da estrutura dos programas de saúde pode levar a subutilização dos mesmos e o aumento das ações judiciais, prejudicando a qualidade da assistência prestada à população ${ }^{14}$. Considerando a complexidade da organização da Assistência Farmacêutica no SUS, foi desenvolvido um sítio gratuito que dissemina informações de medicamentos no SUS. Neste estudo é relatada a experiência no processo de desenvolvimento deste sítio.

\section{Relato de experiência}

O tema do trabalho de conclusão de residência foi escolhido considerando os eixos norteadores dos programas de residência em saúde, destacando-se a importância de promover a integração entre serviço, ensino, pesquisa e comunidade ${ }^{15}$. Sabendo da dificuldade de compreensão da organização dos medicamentos no SUS e por se tratar de um programa de residência farmacêutica, foi selecionado o tema disseminação de informações sobre assistência farmacêutica.

\section{Local de estudo}

Este trabalho foi desenvolvido em um hospital universitário (HU) como trabalho de conclusão de residência de um programa de residência farmacêutica. O trabalho foi realizado no período de Agosto de 2012 a Março de 2014.

A residência farmacêutica tem carga horária de 5.670 horas, com duração de 2 anos, e é estruturada de forma a ensinar ao residente o papel do farmacêutico nas diferentes esferas do cuidado do paciente: atenção primária, hospitalar e vigilância sanitária. Assim, o residente farmacêutico realiza estágio nos seguintes serviços: unidades básicas de saúde (UBS), HU e núcleo de farmacovigilância de um centro de vigilância sanitária.

\section{Identificação do problema}

Durante a residência, verificou-se que os profissionais da saúde e pacientes apresentavam dificuldades no entendimento da organização da Assistência Farmacêutica. Esses problemas foram identificados durante os estágios práticos da residência em UBS (duração de 360 horas), farmacovigilância no HU (duração de 
180 horas) e centro de vigilância sanitária (duração de 180 horas), e, farmácia hospitalar (duração de 224 horas) e farmácia clínica (duração de 3058 horas) no HU. Nestes estágios ocorreu interação entre residente, equipe multiprofissional e pacientes durante as atividades de dispensação de medicamentos na UBS, discussão de casos clínicos no HU e centro de vigilância sanitária, acompanhamento farmacoterapêutico nas unidades de internação, visitas médicas, e orientação de alta de pacientes internados no HU.

A identificação dos problemas foi realizada por meio da observação dos motivos da não dispensação de medicamentos aos pacientes na UBS, dos questionamentos dos profissionais de saúde sobre a forma correta de prescrever medicamentos no SUS e sobre a lista de medicamentos padronizados no SUS. Os problemas identificados foram categorizados conforme apresentado no Quadro 1.

Quadro 1. Problemas de não compreensão da Assistência Farmacêutica que foram identificados durante os estágios da residência.

\begin{tabular}{|l|}
\hline Problemas identificados \\
\hline falta de entendimento das normas da Portaria 344/98* \\
\hline falta de entendimento da diferença entre REMUMEi e RENAMEii \\
\hline ausência de informaç̃̃es quanto aos medicamentos disponíveis na atenção primária \\
\hline não compreensão dos formulários exigidos para certos medicamentos da REMUMEii \\
\hline não compreensão das regras de prescrição de medicamentos no município de São Paulo \\
\hline não entendimento das regras do Componente Especializado \\
\hline
\end{tabular}

(i) RENAME: Relação Nacional de Medicamentos Essenciais. (ii) REMUME: Relação Municipal de Medicamentos. * Portaria 344/98: legislação que aprova o regulamento técnico sobre substâncias e medicamentos sujeitos a controle especial, ou seja, controla o comércio e o uso de entorpecente ou psicotrópico ou outros sujeitos a controle especial. Fonte: Elaborado pelos autores (2017)

\section{Propostas de projeto}

Após a discussão dos problemas identificados (Quadro 1) foram propostas duas opções de projetos: 1) manual impresso sobre a organização da Assistência Farmacêutica no SUS; 2) sítio contendo informações sobre a Assistência Farmacêutica no SUS. Ambas as propostas foram analisadas conforme suas vantagens e desvantagens (Quadro 2).

Quadro 2. Descrição das vantagens e desvantagens das propostas de projeto considerando os problemas identificados.

\begin{tabular}{|c|c|c|}
\hline Propostas & Vantagens & Desvantagens \\
\hline $\begin{array}{l}\text { 1) Manual impresso } \\
\text { da Assistência } \\
\text { Farmacêutica }\end{array}$ & $\begin{array}{l}\text { Facilita a consulta rápida } \\
\text { Útil em situações sem internet disponível } \\
\text { Possibilidade de distribuição nos serviços de } \\
\text { saúde e para os pacientes }\end{array}$ & $\begin{array}{l}\text { Difícil e demorada atualização } \\
\text { Ferramenta estática } \\
\text { Limitada quantidade de informações } \\
\text { Complexa formatação das informações } \\
\text { Limitado público atingido } \\
\text { Necessidade de recursos para impressão } \\
\text { e disseminação }\end{array}$ \\
\hline $\begin{array}{l}\text { 2) Sítio da Assistência } \\
\text { Farmacêutica }\end{array}$ & $\begin{array}{l}\text { Fácil e rápida atualização } \\
\text { Pouca limitação de espaço para inserção de } \\
\text { informações } \\
\text { Ferramenta dinâmica } \\
\text { Simples e rápida formatação das informações } \\
\text { Gratuito } \\
\text { Possibilita que a informação atinja número } \\
\text { grande de pessoas }\end{array}$ & $\begin{array}{l}\text { Necessidade de internet } \\
\text { Necessidade de dispositivo com acesso } \\
\text { a internet }\end{array}$ \\
\hline
\end{tabular}

Fonte: Elaborado pelos autores (2017) 
Considerando as vantagens e desvantagens identificadas (Quadro 2) e principalmente a necessidade de uma ferramenta dinâmica e abrangente de disseminação de informações sobre a Assistência Farmacêutica, com possibilidade de atualização constante, decidiu-se pela criação do sítio. O sítio recebeu o nome de "Residência Farmacêutica", porque, inicialmente, o sítio foi delineado para orientar somente os residentes farmacêuticos. No entanto, devido a riqueza de informações anexadas, optou-se por abrir o acesso para auxiliar maior número de pessoas na compreensão da Assistência Farmacêutica no SUS.

\section{Plataforma de desenvolvimento}

O sítio foi desenvolvido na plataforma Google sites ${ }^{\circledR}$. Esta ferramenta foi escolhida por ser gratuita, de fácil uso e possibilitar a interação com outras ferramentas do Google ${ }^{\circledR}$ (como o Drive e Agenda), permitindo atualização rápida dos documentos e informações inseridas. Além disso, o Google site ${ }^{\circledR}$ tem boa interação com dispositivos eletrônicos como celulares e tablets, facilitando a aceitação pelo público e disseminação das informações do sítio.

\section{Criação do sítio}

\section{Seleção dos temas}

Visto que os problemas encontrados estavam principalmente relacionados com a atenção primária, foram realizadas reuniões com 2 farmacêuticos especialistas em atenção primária para discussão dos temas a serem inseridos no sítio. Ambos farmacêuticos foram escolhidos por possuírem experiência em farmácia clínica, atenção farmacêutica e atuarem em UBS.

Os problemas identificados (Quadro 1) foram discutidos com os especialistas. Todas as sugestões feitas por eles foram seguidas, com exceção da criação de página sobre oncologia, pois os envolvidos no projeto não possuíam experiência suficiente na referida área. Posteriormente, os assuntos selecionados foram validados pela orientadora do projeto. O Quadro 3 apresenta os principais temas incluídos no sítio, que podem ser divididos em 2 grupos: informações sobre a Assistência Farmacêutica no SUS e orientações para a promoção do uso racional de medicamentos.

Quadro 3. Temas incluídos no sítio, a partir da lista de problemas identificados e após discussões com farmacêuticos especialistas.

\section{Temas}

1) informações sobre a Assistência Farmacêutica no SUSi

\section{Tópicos selecionados}

Componentes da Assistência Farmacêutica no SUS: Básico,

Especializado e Estratégico

Programa Farmácia Popular e Dose Certa

Regras para prescrição no SUS, incluindo aquelas para dispensação de psicofármacos e medicamentos de dispensação diferenciada

Medicamentos não disponíveis no SUS

2) orientações para a promoção do uso racional de medicamentos

Uso de medicamentos por idosos

Dose máxima de anti-hipertensivos, hipoglicemiantes orais, psicofármacos

Uso de medicamentos durante a gravidez e amamentação

Diluição de medicamentos intravenosos

Cálculos farmacêuticos

(i) SUS: Sistema Único de Saúde.

Fonte: Elaborado pelos autores (2017) 


\section{Conteúdo do sítio}

Para embasamento das recomendações quanto à prescrição e acesso de medicamentos no SUS foram utilizadas as legislações vigentes, e inseridos links para seu acesso.

As informações oficiais sobre os programas do governo foram obtidas dos seguintes sítios oficiais: Portal da Saúde, Secretaria do Estado da Saúde São Paulo, Secretaria da Saúde do Município de São Paulo, Agência Nacional de Vigilância Sanitária (ANVISA), Fundação para o Remédio Popular (FURP).

As informações técnicas dos medicamentos foram obtidas a partir da busca nas bases de dados: Micromedex ${ }^{\circledR}$, UpToDate $^{\circledR}$, Drugbank $^{\circledR}$ e bulário da ANVISA.

\section{Adequação da linguagem do sítio}

Para possibilitar melhor compreensão pelo público leigo, o conteúdo do sítio foi verificado independentemente por duas pessoas não profissionais de saúde. Todas as sugestões listadas foram aceitas e o texto correspondente foi reescrito.

\section{Avaliação das informações postadas}

Como este sítio é parte de uma monografia de conclusão de residência, todas as informações contidas foram verificadas por 3 docentes farmacêuticos. Todas as sugestões feitas foram incluídas no sítio.

\section{Relevância do sítio criado}

Apesar de não ter sido verificada a opinião dos usuários sobre sítio, por ter ganhado o Prêmio SUS-2014, categoria monografia de especialização ou residência, este trazendo visibilidade para o programa e para o tema informações da Assistência Farmacêutica no SUS.

\section{Discussão}

Neste trabalho relatou-se o processo de desenvolvimento de ferramenta virtual (sítio) que dissemina informações de Assistência Farmacêutica no SUS.

A construção de sítio não é simples e demanda tempo, habilidades específicas e apoio de profissionais de tecnologia da informação. Algumas ferramentas disponíveis na internet facilitaram a criação do sítio (Google sites ${ }^{\circledR}$ e vídeos do YouTube ${ }^{\circledR}$ ), no entanto as seguintes dificuldades foram encontradas: obtenção de informações sobre assistência farmacêutica e acesso aos medicamentos no SUS, ausência de suporte de tecnologia de informação e de desenhos gráficos para criação de figuras próprias para o sítio, tempo limitado para entrega da monografia, desafio em encontrar informações sobre assistência farmacêutica em sítios oficiais, ausência de recursos financeiros para subsídio do projeto.

Apesar das dificuldades, o sítio criado possui uma importante vantagem em relação aos demais sítios oficiais: as informações da Assistência Farmacêutica em só um local independentemente do nível de atenção à saúde. Nos sítios oficiais os principais problemas encontrados foram: necessidade de abrir diversas páginas até encontrar a lista de medicamentos padronizados ou formulários específicos para dispensação de medicamentos especializados, muitas das informações encontravam-se desatualizadas, e frequentemente o sítio do Ministério da Saúde encontrava-se em manutenção. Estudo prévio identificou que sítios oficiais apresentam informações desatualizadas sobre a Assistência Farmacêutica e poucas informações que facilitem o acesso aos medicamentos ${ }^{16}$. Recentemente o problema dos sítios oficiais tem ganhado destaque entre gestores do SUS. Em seminário do Conselho Nacional de Secretários de Saúde (CONASS Debate) foi apontado que os usuários do SUS reclamam da desorganização no fluxo de informações dos sítios oficiais ${ }^{17}$. 
Neste debate são listados os problemas frequentes que também foram detectados nos sítios oficiais: ficam fora do ar, ausência de facilitadores de navegação para usuários leigos, presença de links que levam a páginas inexistentes ${ }^{17}$. Uma das causas da recorrência destes problemas pode estar relacionada ao pouco investimento em comunicação focada no usuário ${ }^{17}$.

Em contraste aos problemas encontrados para a criação do sítio, algumas facilidades possibilitaram são destacadas: acompanhamento e discussões com farmacêuticos especialistas em atenção primária e com docente, auxílio e opiniões dos farmacêuticos e residentes do HU, disponibilidade de acesso à internet e as bases de dados com acesso restrito, como UpToDate ${ }^{\circledR}$.

Das vantagens do sítio criado destaca-se a confiabilidade das informações disponibilizadas. Todo o conteúdo inserido foi baseado em legislações vigentes, bases de dados consagradas internacionalmente (como o UpToDate ${ }^{\circledR}$ ), além de ter sido avaliado por farmacêuticos especialistas da atenção primária e por docentes. O conteúdo disponibilizado abrange temas relacionados à promoção do uso racional e seguro de medicamentos: dose máxima de medicamentos, quadros de diluição, medicamentos inapropriados para idosos, classificação de risco de medicamentos para gestantes e lactantes e, cálculos farmacêuticos. Ao contrário do relatado em revisão prévia de sítios brasileiros, nos quais foram constatadas disseminações de informações incorretas, incompletas, desatualizadas e com pouca base científica ${ }^{18}$.

Além disso, o conteúdo engloba informações sobre os componentes da Assistência Farmacêutica no SUS e as regras para o acesso aos medicamentos em um único espaço virtual - independentemente do componente (especializado, estratégico ou básico) e programa (Dose Certa e Farmácia Popular) no qual o medicamento está disponível. O problema da fragmentação da informação em saúde dos sítios oficiais tem sido relatado na literatura. Em debate recente do CONASS apontou-se que muitas das informações de saúde transmitidas pela mídia apresentam distorções e fragmentação, sendo um desafio explicar a população a dinâmica e estrutura SUS ${ }^{17}$.

Outro aspecto relevante é a imagem positiva que o sítio criado traz do SUS uma vez que apresenta todas as opções de medicamentos disponíveis no sistema público de saúde, não abordando o SUS como normalmente é mostrado na mídia, como algo ruim e que não funciona adequadamente ${ }^{17}$. Em vez desta visão negativa, mostra como o SUS tem diversos programas e ainda traz os caminhos oficiais para obtenção de medicamentos não padronizados nas listas do SUS, sem a necessidade de ações judiciais. Sabendo que as tecnologias foram incorporadas no SUS após estudos e discussões entre especialistas da área e que muitas decisões judiciais ignoram as alternativas terapêuticas disponíveis no SUS ${ }^{19-21}$, orientar a população e profissionais de saúde quanto os medicamentos disponíveis e o procedimento para solicitação dos não disponíveis no SUS é crucial no uso racional dos recursos em saúde.

Uma limitação deste trabalho é a não avaliação formal da opinião dos usuários. Durante todo o processo de criação do sítio as sugestões de leigos em saúde e dos farmacêuticos residentes do HU foram consideradas. Porém, não foi o objetivo deste trabalho quantificar e avaliar estas sugestões. Além disso, a importância do sítio criado foi reconhecida nacionalmente por meio da obtenção do primeiro lugar no Prêmio de Incentivo e Inovação para o SUS - 2014, categoria monografia de residência. Outra limitação do sítio é a não inclusão de todos os programas do SUS. No entanto, o sítio é dinâmico e o conteúdo pode ser atualizado e novas páginas podem ser criadas conforme a demanda e a evolução dos programas do SUS.

Sabendo que a interneté uma poderosa ferramenta comunicadora em saúde ${ }^{22}$, principalmente no contexto de promoção da saúde ${ }^{23,24}$, sítios de saúde com boa qualidade são importantes tanto para profissionais de saúde quanto para a população geral, ajudando-os a entender a organização do sistema público de saúde, aprender sobre os programas de medicamentos e seus direito, e melhorar a relação profissional-paciente ${ }^{25}$. No entanto, a dificuldade em obter informações corretas de medicamentos na Internet, especialmente em português e sobre as particularidades do SUS, prejudica a promoção e educação em saúde. Assim, o sítio criado tem potencial para promover a democratização da informação de saúde, disseminando de forma gratuita conteúdo confiável relacionado a medicamentos. 


\section{Considerações finais}

Este é o primeiro estudo que relata o desenvolvimento de um sítio como um trabalho de conclusão de residência. Assim, este trabalho traz informações importantes que podem ser exemplos para futuros trabalhos de conclusão de residência e/ou criação de sítios com temas relevantes para a saúde no Brasil. Além disso, ressalta-se que foi a partir da observação e discussão dos problemas relacionados à dificuldade de compreensão da Assistência Farmacêutica no SUS que foi possível a criação de um sítio.

\section{Referências}

1. $\quad$ Brasil. Constituição da República Federativa do Brasil (1988). Brasília, DF, Brasil: Senado Federal; 1988.

2. Brasil. Lei n.8080, de 19 de setembro de 1990. Dispõe sobre as condições para a promoção, proteção e recuperação da saúde, a organização e o funcionamento dos serviços correspondentes e dá outras providências. Brasil: Diário Oficial da União; 1990.

3. Vieira FS. Assistência farmacêutica no sistema público de saúde no Brasil. Rev Panam Salud Publica. 2010;27(2):149-56.

4. Carias CM, Vieira FS, Giordano C V , Zucchi P. Medicamentos de dispensação excepcional: histórico e gastos do Ministério da Saúde do Brasil. Rev Saude Publica. 2011;45(2):233-40.

5. Vieira FS, Zucchi P. Financing of Pharmaceutical Services in Brazilian Public Health System. Saude Soc. 2013;22(1):73-84.

6. Aguiar G, Júnior LA da S, Ferreira MAM. Ilegibilidade e ausência de informação nas prescrições médicas: fatores de risco relacionados a erros de medicação. Rev Bras Prom Saude. 2006;19(2):84-91.

7. Guzatto $P$, Bueno D. Análise de prescrições medicamentosas dispensadas na farmácia de uma unidade básica de saúde de Porto Alegre - RS. Rev HCPA. 2007;27(3):20-6.

8. Laste G, Torres IL da S, Deitos A, Souza AC de, Souza A de, Kauffmann C, et al. Análise de prescrições médicas dispensadas em farmácia no Sistema Único de Saúde. Rev HCPA. 2013;33(1):15-25.

9. Lins B de G, Cazzamalli F, Zancanaro V. Análises de erros nas prescrições médicas de uma unidade básica de saúde de um município do meio oeste catarinense. RIES. 2012;1(2):62-77.

10. Silva ERB da, Bandeira VAC, Oliveira KR De. Avaliação das prescrições dispensadas em uma farmácia comunitária no município de São Luiz Gonzaga - RS. Rev Ciênc Farm Básica Apl. 2012;33(2):275-81.

11. Valadão AF, Firmino KF, Moreira AL de P, Castro GD, Loures GF, Públio RN, et al. Estudo das prescrições de medicamentos nas Unidades Básicas de Saúde de Coronel Fabriciano - MG, quanto aos preceitos legais. Rev Bras Farm. 2008;89(4):298-301.

12. Júnior DP de L, Amaral RT do, Abriata JP, Pelá IR. A satisfação como resultado de um programa de atenção farmacêutica para pacientes idosos, em Ribeirão Preto (SP) - Brasil. Seguim Farm. 2005;3(1):30-42.

13. Melo DO De, Ribeiro E, Storpirtis S. A importância e a história dos estudos de utilização de medicamentos. Braz J Pharm Sci. 2006;42(4):475-85.

14. Vieira FS, Zucchi P. Distorções causadas pelas ações judiciais à política de medicamentos no Brasil. Rev Saude Publica. 2007;41(2):214-22.

15. Brasil. Ministério da Educação. Ministério da Saúde. Portaria Interministerial no 1.077, de 12 de novembro de 2009. Dispõe sobre a Residência Multiprofissional em Saúde e a Residência em Área Profissional da Saúde e institui o Programa Nacional de Bolsas para Residências Multiprofissionais e em Área Profiss. 2009.

16. Gonçalves RAM. Avaliação das informações sobre Assistência Farmacêutica em sítios governamentais [Internet]. Universidade Federal do Rio Grande do Sul. 2012 [cited 2015 Jul 16]. p. 38. Available from: http://hdl.handle.net/10183/67803

17. Brasil. Conselho Nacional de Secretários de Saúde. CONASS Debate - Que saúde você vê? 4th ed. Conselho Nacional de Secretários de Saúde, editor. Brasília: CONASS; 2015. 76 p. 
18. Malafaia G, Castro AL da S, Rodrigues AS de L. A qualidade das informações sobre doenças disponíveis em websites brasileiros: uma revisão. Arq bras ciênc saúde. 2011;36(2):72-8.

19. Fonseca EM da, Costa N do R. Federalismo, complexo econômico-industrial da saúde e assistência farmacêutica de alto custo no Brasil. Cien Saude Colet. 2015;20(4):1165-76.

20. Pepe VLE, Figueiredo T de A, Simas L, Osorio-de-Castro CGS, Ventura M. A judicialização da saúde e os novos desafios da gestão da assistência farmacêutica. Cien Saude Colet. 2010;15(5):2405-14.

21. Lima-Dellamora E da C, Caetano R, Osorio-de-Castro CGS. Dispensação de medicamentos do componente especializado em polos no Estado do Rio de Janeiro. Cien Saude Colet. 2012;17(9):2387-96.

22. Koch-Weser S, Bradshaw YS, Gualtieri L, Gallagher SS. The Internet as a health information source: findings from the 2007 Health Information National Trends Survey and implications for health communication. J Heal Commun. 2010;15(Suppl 3):279-93.

23. Coleman JJ, Mcdowell SE. An agenda for UK clinical pharmacology: The potential of the internet. $\mathrm{Br}$ J Clin Pharmacol. 2012;73(6):953-8.

24. Cassell MM, Jackson C, Cheuvront B. Health communication on the Internet: an effective channel for health behavior change? J Heal Commun. 1998;3(1):71-9.

25. Sommerhalder K, Abraham A, Zufferey MC, Barth J, Abel T. Internet information and medical consultations: Experiences from patients' and physicians' perspectives. Patient Educ Couns. $2009 ; 77(2): 266-71$. 